\title{
Serum NMR metabolomics to differentiate haematologic malignancies
}

\author{
Wojciech Wojtowicz ${ }^{1}$, Angelika Chachaj², Andrzej Olczak ${ }^{3}$, Adam Ząbek ${ }^{1}$, Elżbieta \\ Piątkowska ${ }^{4}$, Justyna Rybka ${ }^{5}$, Aleksandra Butrym ${ }^{6,7}$, Monika Biedroń5, Grzegorz \\ Mazur ${ }^{6}$, Tomasz Wróbel ${ }^{5}$, Andrzej Szuba ${ }^{2}$ and Piotr Młynarz ${ }^{1}$ \\ ${ }^{1}$ Wroclaw University of Technology, Department of Bioorganic Chemistry, Wroclaw, Poland \\ ${ }^{2}$ Wroclaw Medical University, Department of Angiology, Wroclaw, Poland \\ ${ }^{3}$ Opole University of Technology, Faculty of Electrical Engineering, Automatic Control and Informatics, Opole, Poland \\ ${ }^{4}$ Wrocław Research Centre EIT+, Wroclaw, Poland \\ ${ }^{5}$ Department of Haematology, Blood Neoplasms, and Bone Marrow Transplantation, Wroclaw Medical University, Wroclaw, \\ Poland \\ ${ }^{6}$ Department of Internal Medicine, Wroclaw Medical University, Wroclaw, Poland \\ ${ }^{7}$ Department of Physiology, Wroclaw Medical University, Wroclaw, Poland \\ Correspondence to: Piotr Młynarz, email: piotr.mlynarz@pwr.edu.pl \\ Keywords: metabolomics; haematological malignancies; $\mathrm{nHL} ; \mathrm{AML} ; \mathrm{CLL}$ \\ Received: January 24, $2018 \quad$ Accepted: April 07, $2018 \quad$ Published: May 11, 2018 \\ Copyright: Wojtowicz et al. This is an open-access article distributed under the terms of the Creative Commons Attribution License \\ 3.0 (CC BY 3.0), which permits unrestricted use, distribution, and reproduction in any medium, provided the original author and \\ source are credited.
}

\section{ABSTRACT}

Haematological malignancies are a frequently diagnosed group of neoplasms and a significant cause of cancer deaths. The successful treatment of these diseases relies on early and accurate detection. Specific small molecular compounds released by malignant cells and the simultaneous response by the organism towards the pathological state may serve as diagnostic/prognostic biomarkers or as a tool with relevance for cancer therapy management. To identify the most important metabolites required for differentiation, an ${ }^{1} \mathrm{H}$ NMR metabolomics approach was applied to selected haematological malignancies. This study utilized 116 methanol serum extract samples from AML $(n=38), n H L(n=26), \operatorname{CLL}(n=21)$ and HC $(n=31)$. Multivariate and univariate data analyses were performed to identify the most abundant changes among the studied groups. Complex and detailed VIP-PLS-DA models were calculated to highlight possible changes in terms of biochemical pathways and discrimination ability. Chemometric model prediction properties were validated by receiver operating characteristic (ROC) curves and statistical analysis. Two sets of eight important metabolites in HC/AML/ $\mathrm{CLL} / \mathrm{nHL}$ comparisons and five in AML/CLL/ $\mathrm{nHL}$ comparisons were selected to form complex models to represent the most significant changes that occurred.

\section{INTRODUCTION}

Haematological malignancies are clonal diseases. Neoplasms of the haematopoietic system are derived from myeloid lineage stem or progenitor cells, while the tumours of the lymphatic system originate from lymphoid lineage precursor or mature cells [1]. The factors causing these malignant transformation disorders are both genetic and environmental. They lead to abnormal signal transduction and gene expression in the cell and to disturbances during key haematopoiesis processes, such as self-renewal, proliferation and differentiation [1]. According to the Polish National Cancer Registry, the number of new cases of haematological malignancies increased near 2-fold in the past three decades. In 1990 , the incidence rate was estimated as $8.8 / 100000$ (10.4 for men and 7.4 for women), and in 2010 it was $16.8 / 100000$ inhabitants (18.1 for men and 15.5 for 
women). An important risk factor for haematological disorder is the patient's age. The one of the most frequent haematological disorders, which occur in adults, are acute myeloid leukaemia (AML) in the haematopoietic system, non-Hodgkin's lymphoma (nHL) and chronic lymphocytic leukaemia (CLL) among lymphatic system neoplasms. Most cases (approximately 60\%) are recorded between 50 and 79 years of age $[2,3,4]$. The 5-year survival rates among these haematological malignancies are $27 \%$ for $\mathrm{AML}, 70 \%$ for $\mathrm{nHL}$ and $83 \%$ for CLL patients $[1,3,4]$. The early diagnosis and effective treatment of haematological malignancies continues to be an overwhelming challenge. Malignant cells exhibit a distinct metabolic phenotype, which may be reflected by the release of low molecular weight compounds $[5,6]$. The analysis of changes in these compounds may serve as a diagnostic/prognostic biomarkers not only for the malignancies but also as a tool with relevance to cancer management, therapy and monitoring [7]. Recently, numerous studies have characterized the metabolic profiles of a variety of malignant tumors, including brain, lung, prostate, pancreatic, breast, ovarian, liver and thyroid $[8,9]$.

The purpose of the study was to determine the metabolic profiles of the three common haematological malignancies in adults, acute myeloid leukaemia (AML), non-Hodgkin's lymphoma (nHL) and chronic lymphocytic leukaemia (CLL), using an metabolomics approach, for highlighting potential important influence on organism metabolism caused by haematological malignancies and indication possible biomarkers.

\section{RESULTS}

Altogether, 50 metabolites were assigned (Figure 1 and Supplementary Table 1) and 18 resonance signals were marked as unknown. From the identified metabolites, 45 and the 18 unknown signals were used for the chemometric models due to their non-overlapping signals. The found metabolites exhibited different trends in the particular comparisons, such as only increasing, only decreasing and with variate trends (Supplementary Table 2).

The obtained chemometric models, which were based on VIP-PLS-DA, could classify patients' haematological malignancy samples into individual groups versus the healthy control group. Comparisons between the individual haematologic malignancies were also based on VIP-PLS-DA models (calculated separately) and showed lower model parameter values than comparisons with healthy individuals (Table 1). In general, five of the eight models passed the validation test, though most of these models did not include CLL.

\section{Analysis of all the studied groups (AML, CLL, nHL, and HC)}

The analysis of the VIP-PLS-DA models calculated for three malignancies units and control group using a set of metabolites with the greatest discriminatory potential (Figure 2A and 2B). This pool of metabolites included (sequentially decreasing VIP values) $O$-phosphocholine, glutamine, phenylalanine, tryptophan, glutamate,

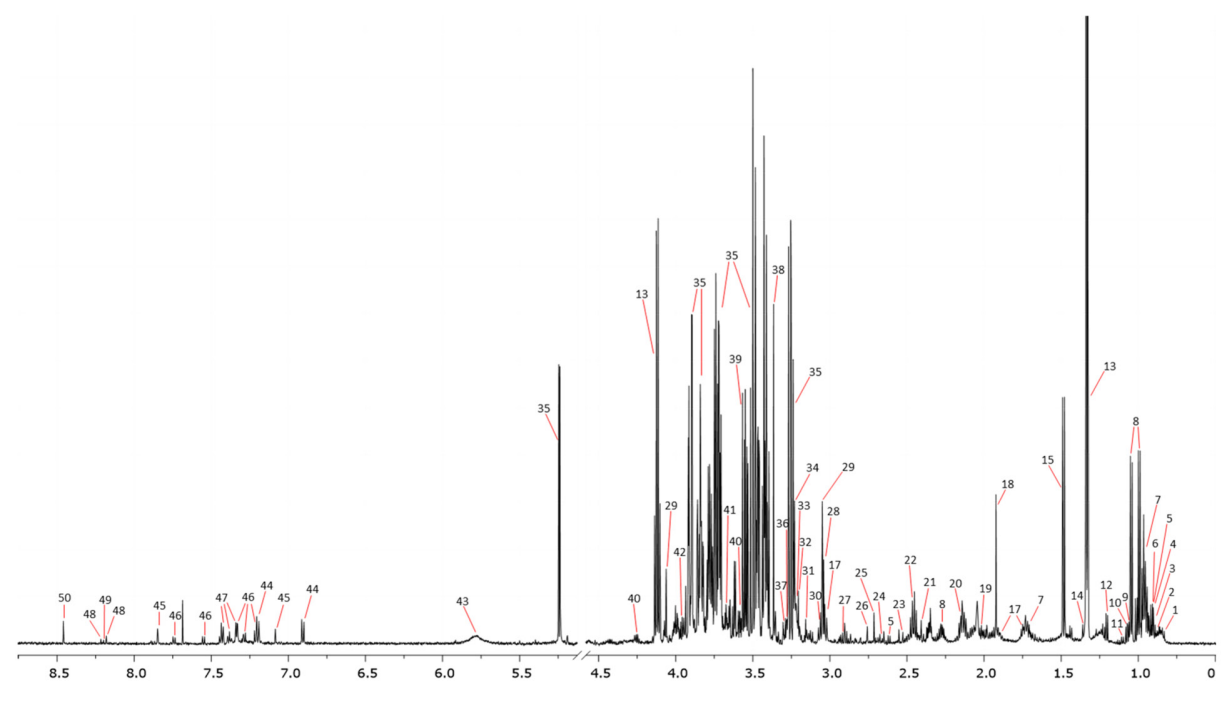

Figure 1: Representative ${ }^{1}$ H NMR spectrum with marked resonance signals. 1 - 2-Hydroxyisovalerate, 2 - 2-Hydroxybutyrate, 3 - Isovalerate, 4 - Isoleucine, 5 - 2-Oxoisocaproate, 6 - Alloisoleucine, 7 - Leucine, 8 - Valine, 9 - Isobutyrate, 10 - 2-Methylglutarate, 11 - 3-Methyl-2-oxovalerate, 12 - 3-Hydroxybutyrate, 13 - Lactate, 14 - 2-Hydroxyisobutyrate, 15 - Alanine, 17 - Lysine, 18 - Acetate, 19 - Proline, 20 - Glutamine, 21 - Succinate, 22 - Glutamate, 23 - Citrate, 24 - Methionine, 25 - Dimethylamine, 26 - Sarcosine, 27 - N,NDimethylglycine, 28 - Creatine, 29 - Creatinine, 30- Ornithine, 31 - Dimethyl sulfone, 32 - Choline, 33 - O-Phosphocholine, 34 - sn-Glycero3-phosphocholine, 35 - Glucose, 36 - Betaine, 37 - Taurine, 38 - Methanol, 39 - Glycine, 40 - Threonine, 41 - Glycerol, 42 - Serine, 43 - Urea, 44 - Tyrosine, 45 - Histidine, 46 - Tryptophan, 47 - Phenylalanine, 48 - Hypoxanthine, 49 - Oxypurinol, and 50 - Formate. 
Table 1: The VIP-PLS-DA model parameters for each comparison based on serum samples

\begin{tabular}{|c|c|c|c|c|c|c|c|}
\hline Comparison & $\begin{array}{c}\text { Latent } \\
\text { variables }\end{array}$ & $\mathbf{R}^{2} \mathbf{X}(\mathbf{c u m})$ & $\mathbf{R}^{2} \mathbf{Y}(\mathbf{c u m})$ & $Q^{2}$ (cum) & CV-ANOVA & $\begin{array}{c}\text { AUC } \\
\text { training }\end{array}$ & AUC test \\
\hline $\begin{array}{l}\text { HC/AML/CLL/ } \\
\text { nHL }\end{array}$ & 2 & 0.370 & 0.267 & 0.223 & $3.96 \mathrm{E}-12$ & - & - \\
\hline HC /AML & 2 & 0.512 & 0.864 & 0.744 & $3.18 \mathrm{E}-05$ & 1.000 & 0.975 \\
\hline HC/CLL & 2 & 0.472 & 0.662 & 0.248 & $1.05 \mathrm{E}-01$ & 0.852 & 0.588 \\
\hline HC/nHL & 2 & 0.614 & 0.622 & 0.37 & $1.08 \mathrm{E}-02$ & 0.898 & 0.853 \\
\hline AML/CLL/nHL & 2 & 0.366 & 0.394 & 0.315 & $4.98 \mathrm{E}-11$ & - & - \\
\hline AML/nHL & 2 & 0.530 & 0.692 & 0.575 & 7.74E-04 & 0.929 & 0.837 \\
\hline AML/CLL & 2 & 0.459 & 0.719 & 0.383 & $6.20 \mathrm{E}-02$ & 0.879 & 0.932 \\
\hline CLL/nHL & 2 & 0.582 & 0.469 & 0.287 & $7.84 \mathrm{E}-02$ & 0.837 & 0.655 \\
\hline
\end{tabular}

histidine, formate, 2-hydroxyisovalerate, citrate, snglycero-3-phosphocholine, ornithine, 2-oxoisocaproate, 2-hydroxybutyrate, alanine, 2-methylglutarate, betaine, sarcosine, creatine, acetate, proline, and choline.

The loading plot (Figure 2B) of comprehensive model allows for metabolite selections and verification, which had the most important contribution to the differentiation between investigated groups. From the determined metabolites, three of the most important for differentiation among the identified and unidentified compounds based on the closest distance to Y-variable have been selected for the $\mathrm{HC}$ vs. AML vs. CLL vs. nHL comparison $\left(\mathrm{R}^{2} \mathrm{X}=0.401, \mathrm{Q}^{2}\right.$ $=0.218$ ). Hence, the following sets of identified compounds were chosen: (1) for $\mathrm{HC}$ - histidine, $O$-phosphocholine, and glutamine; (2) for AML - phenylalanine, glutamate and formate; (3) for CLL - choline, alanine, and proline and (4) for nHL- creatine, sarcosine, and acetate. Among the unidentified metabolites, unk_4 was important for $\mathrm{HC}$, unk_16 for AML, and unk_2 for $\bar{n} \overline{H L}$, while no unidentified metabolites were important for CLL. Moreover, using a single metabolite in multiple groups was avoided. The introduced general comparisons shown in Figure 2A and 2B demonstrate the ability to distinguish between the investigated groups, though it does not accurately reveal the metabolite variation between the specific particular comparisons, e.g., HC vs. AML. Therefore, more detailed chemometric models based on comparisons between two groups were calculated (Table 1 and Figures 3 and 6). The best classification was achieved for the comparison between the $\mathrm{HC}$ and AML groups. This model obtained the greatest ROC curve $\left(\mathrm{AUC}_{\text {training }} 1.00 ; \mathrm{AUC}_{\text {test }} 0.975\right)$ and basic parameter values among the models (Table 1 and Figures 3A and $3 \mathrm{~B}$ ). In contrast, assessment of HC and CLL groups showed the lowest model parameters values $\left(\mathrm{AUC}_{\text {training }}\right.$ 0.852; $\mathrm{AUC}_{\text {test }}$ 0.588) among models based on the HC vs. haematologic malignancy group comparisons. However, the separation between the selected groups was still observed.
A set of 10 identified metabolites were determined for the $\mathrm{HC}$ vs. AML model $\left(\mathrm{R}^{2} \mathrm{X}=0.512, \mathrm{Q}^{2}=0.744\right)$ (Figure 3A) - 2-hydroxybutyrate, 2-methylglutarate, 3-methyl-2-oxovalerate, betaine, formate, glutamate, $O$-phosphocholine, phenylalanine, tryptophan, and histidine were statistically significant and had major contributions to the differentiation based on their VIP values (Supplementary Table 2). Considering the same steps as in the complex model (Figure 2A and 2B), nine relative integrals from unspecified resonances signals, including unk 3, unk_4, unk_6, unk_7, unk_9, unk_10, unk_11, unk_12, and unk_16, were also selected. In the $\mathrm{HC}$ vs. $\mathrm{nHL}$ discrimination model $\left(\mathrm{R}^{2} \mathrm{X}=0.614, \mathrm{Q}^{2}=\right.$ 0.37 ) (Figure $3 \mathrm{~B}$ ), four of the resonances were determined within the relative integral values. Two of the resonances were identified as formate and $O$-phosphocholine and the two unassigned specific metabolites were marked as unk_4 and unk_7. In the midst of all the studied haematological malignancies, the CLL group showed the lowest separation vs. the $\mathrm{HC}$ group $\left(\mathrm{R}^{2} \mathrm{X}=0.472, \mathrm{Q}^{2}\right.$ $=0.248$ ) (Figure 3C) based on the VIP-PLS-DA model. Despite of low separation, 4 identified metabolites and 4 unknowns were determined, including dimethylamine, formate, glutamate, N,N-dimethylglycine, unk_3, unk_6, unk_7, and unk_9, which significantly affected the discrimination between the investigated groups.

Statistical analysis performed for all found compounds based on median or mean levels showed that six metabolites were statistically significant $(\mathrm{M}-\mathrm{W}$ or $t$ test) in each of three comparisons between $\mathrm{HC}$ and the haematological malignancies (Figure 4).

\section{Analysis of the haematological malignancy groups (AML, CLL, and $\mathrm{nHL}$ )}

The complex comparisons between the studied haematological cancers were based on a chemometric 

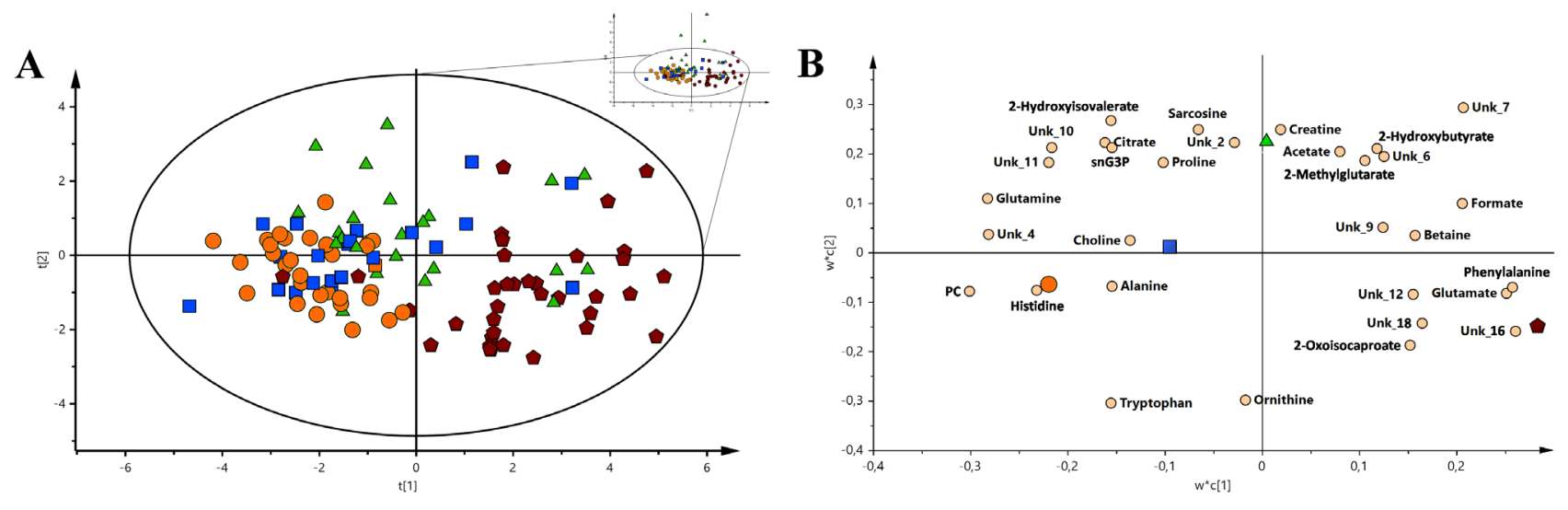

Figure 2: The VIP-PLS-DA models for all the groups used in the study. For a better representation, only Hotelling's T2 range is shown. (A) The PLS-DA general model with all the sample groups. (B) Loading plot of the VIP-PLS-DA model for all groups and samples used in the study. Red pentagon - AML; Orange circle - HC; Green triangle - nHL and Blue box - CLL. The inserted reduced figure serves to show outliers related to the variability in the studied groups.
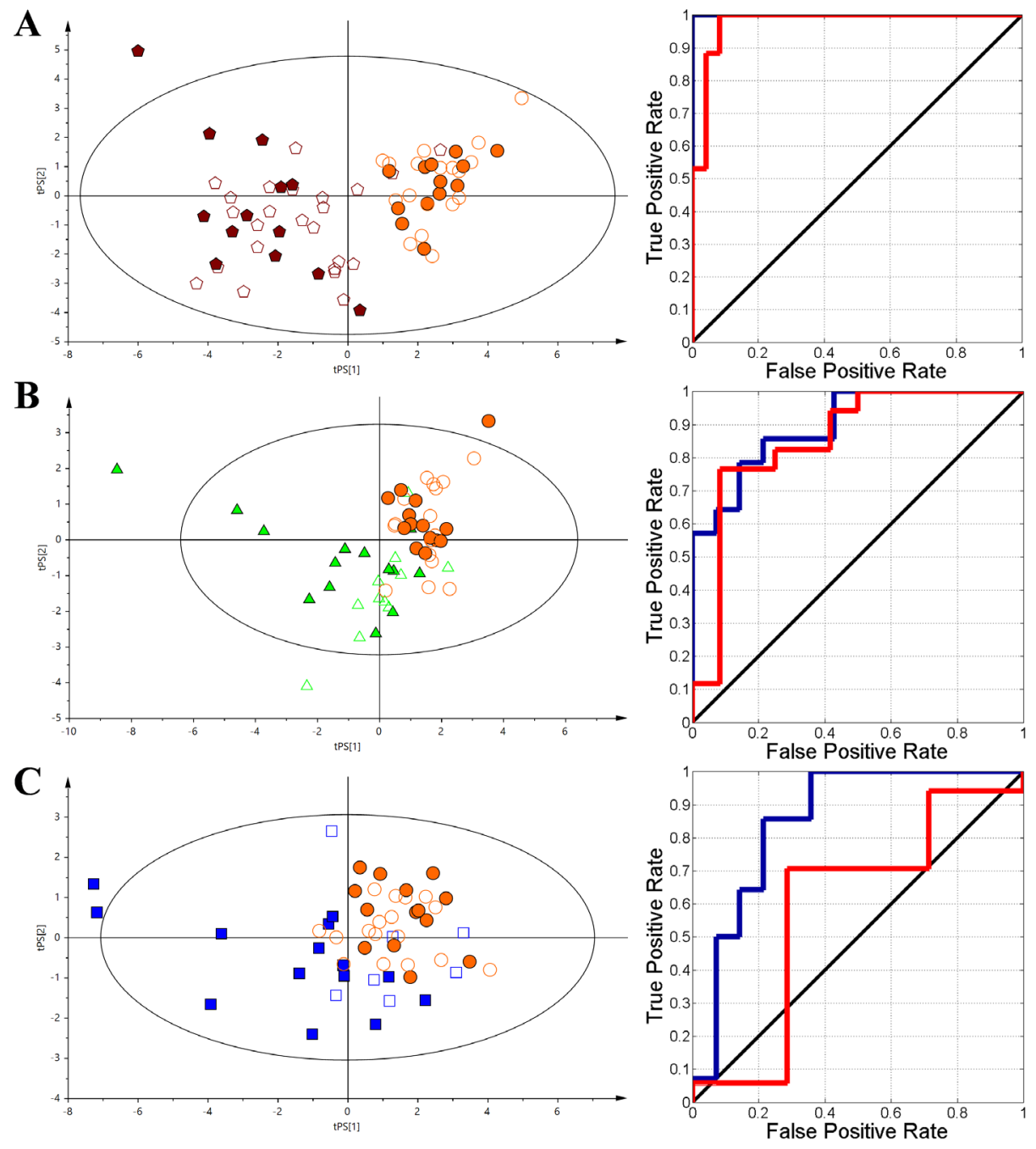

Figure 3: The VIP-PLS-DA models plots with the training (no fill) and test (fill) set samples and ROC curves. The dark blue curve represents the training set and the red curve represents the test set. (A) - HC vs AML; (B) - HC vs nHL, (C) - HC vs CLL. Red pentagon - AML; Orange circle - HC; Green triangle - nHL and Blue box - CLL. 
VIP-PLS-DA model $(\mathrm{R} 2 \mathrm{X}=0.366, \mathrm{Q} 2=0.315)$ (Figure $5 \mathrm{~A}$ and $5 \mathrm{~B})$.

The collective comparison of the three diseases with the most important metabolites were arranged by their decreasing VIP values from the VIPPLS-DA models in the following order: histidine, $O$-phosphocholine, glutamine, 2-oxoisocaproate, citrate, sarcosine, phenylalanine, ornithine, taurine, tryptophan, 2-hydroxyisovalerate, choline, lysine, glutamate, 3-methyl-2-oxovalerate, sn-glycero-3-phosphocholine, threonine, glucose, and proline. The unknowns resonance signals that were important in this comparison were unk_10, unk_4, unk_16, unk_11, unk_15, and unk_13. Among the metabolites, three based on loading plots were selected for each group. The following metabolites were important for the differentiation between the studied groups (Figure 5B): (1) AML group - phenylalanine, glutamate, and 2-oxoisocaproate; CLL group - choline, histidine, and $O$-phosphocholine; and (3) nHL - sarcosine, 2-hydroxyisovalerate, and sn-glycero-3-phosphocholine (Figure 5B).

Three separate chemometric models were calculated to determine accurate differences between the specific cancer groups. The metabolites that were statistically
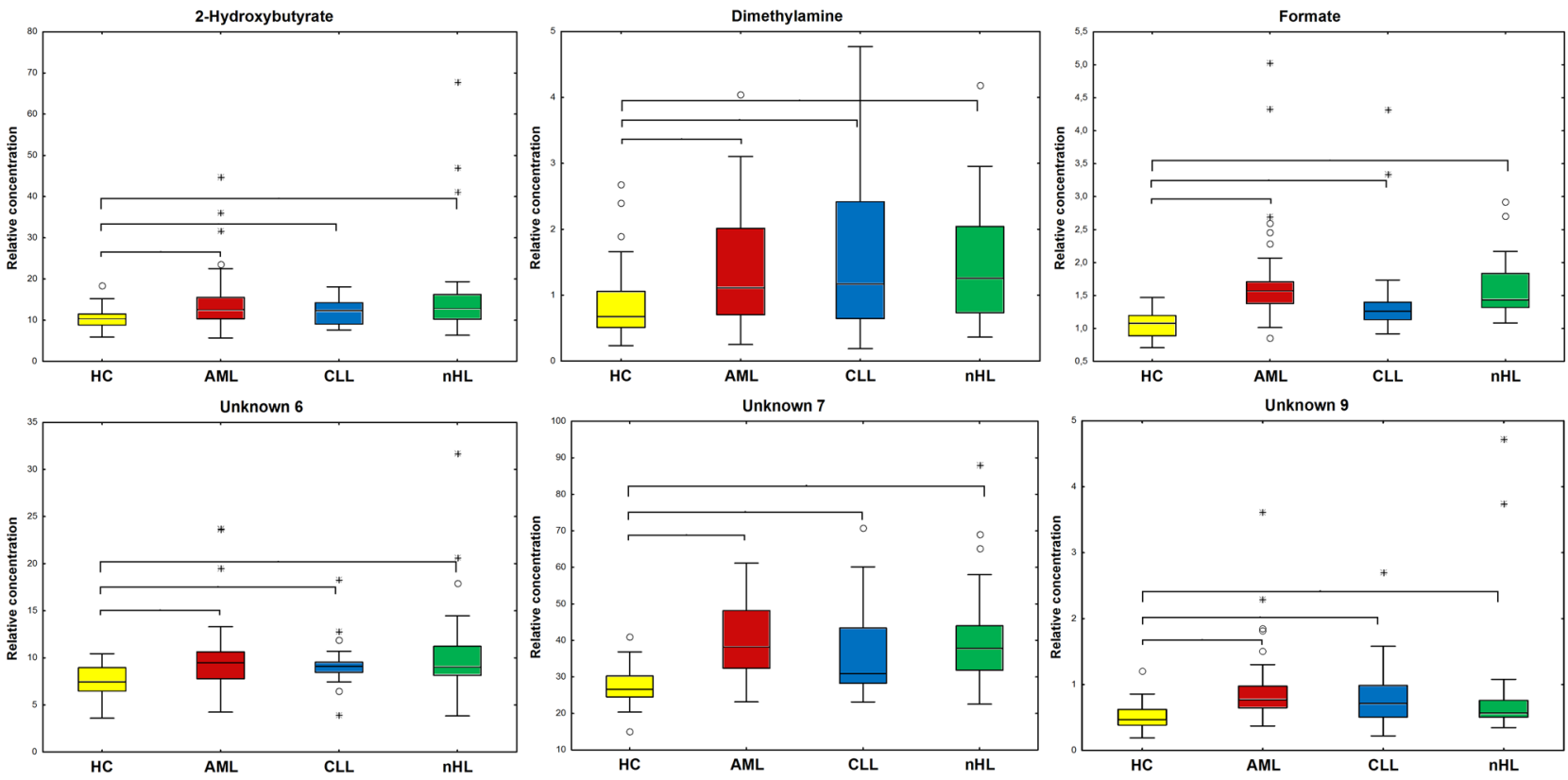

Figure 4: Boxplots of the statistically important metabolites $(p<0.05)$ between $\mathrm{HC}$ and the haematological cancers. Braces mark the comparisons where the selected metabolites are statistically important. Whiskers - non-outlier min-max range; ${ }^{*}$ - extreme; $\circ$ - outlier; bar - median; box - Q1-Q3 interquartile range; yellow - HC; red - AML; blue - CLL; and green - nHL.
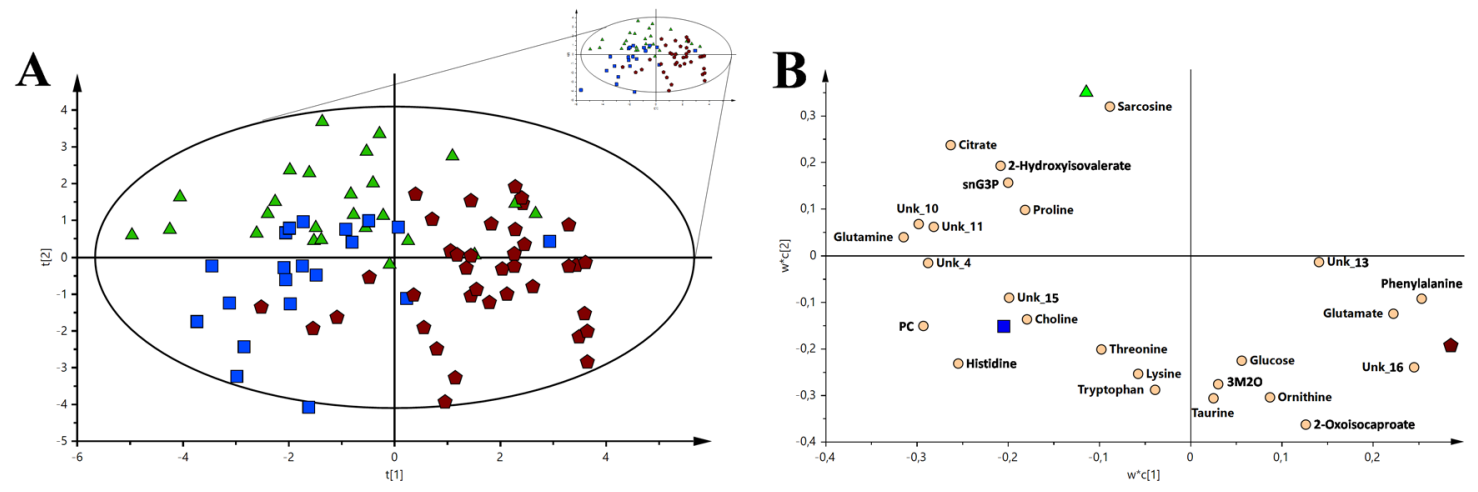

Figure 5: The VIP-PLS-DA models for all the groups used in study. For a better representation, only Hotelling's T2 range is shown. (A) The VIP-PLS-DA model with the haematological malignancy groups. (B) VIP-PLS-DA loading plot for the haematological malignancy groups. Red pentagon - AML; Green triangle - nHL and Blue box - CLL. The inserted reduced figure serves to show outliers related to the variability in the studied groups. 
significant and present in the VIP-PLS-DA model after the first iteration are shown in Supplementary Table 2.

Eight identified metabolites and 4 unknowns were used in the AML vs. CLL comparison $\left(\mathrm{R}^{2} \mathrm{X}=\right.$ $\left.0.459, \mathrm{Q}^{2}=0.383\right)$; phenylalanine, citrate, glutamine, $O$-phosphocholine, dimethyl sulfone, histidne, glutamate, choline, unk_11, unk_10, unk_7, and unk_18 are sorted in a descending order based on their VIP values (Supplementary Table 2 and Figure 6A). For the AML vs. nHL comparison $\left(\mathrm{R}^{2} \mathrm{X}=0.530, \mathrm{Q}^{2}=0.575\right)$, glutamine, 2-oxoisocaproate, citrate, proline, sn-glycero-3phosphocholine, 2-hydroxyisovalerate, unk_10, unk_11, and unk_16 (Supplementary Table 2 and Figure 6B) were selected, while glucose, $O$-phosphocholine, and tryptophan were used for the CLL vs. nHL comparison $\left(\mathrm{R}^{2} \mathrm{X}=0.582, \mathrm{Q}^{2}=0.287\right.$ ) (Supplementary Table 2 and Figure 6C).

Among the differentiating metabolites, compounds that were statistically significant in at least two comparisons between the haematological cancers groups in M-W or $T$ tests (dependent on Shapiro Wilk test results) are presented in Figure 7.

\section{Pathway analysis}

Pathway analysis was performed on the overall data, which contained the HC, AML, CLL, and nHL groups, and indicated the importance of the most changed biochemical pathways. The determined major changes are associated with the following pathways (Impact $>0.1$; p Holm adjust $<0.05$ ): (1) D-glutamine and D-glutamate metabolism, (2) histidine metabolism, (3) alanine aspartate and glutamate metabolism, (4) arginine and proline metabolism, (5) glyoxylate and dicarboxylate metabolism, (6) aminoacyl-tRNA biosynthesis, (7) methane metabolism, (8) phenylalanine metabolism, (9) glycine serine and threonine metabolism, and (10) tryptophan metabolism.

\section{DISCUSSION}

\section{Clinical significance}

Haematological malignancies are a frequently diagnosed group of neoplasms and a significant cause of death induced by cancer $[13,14]$. The successful
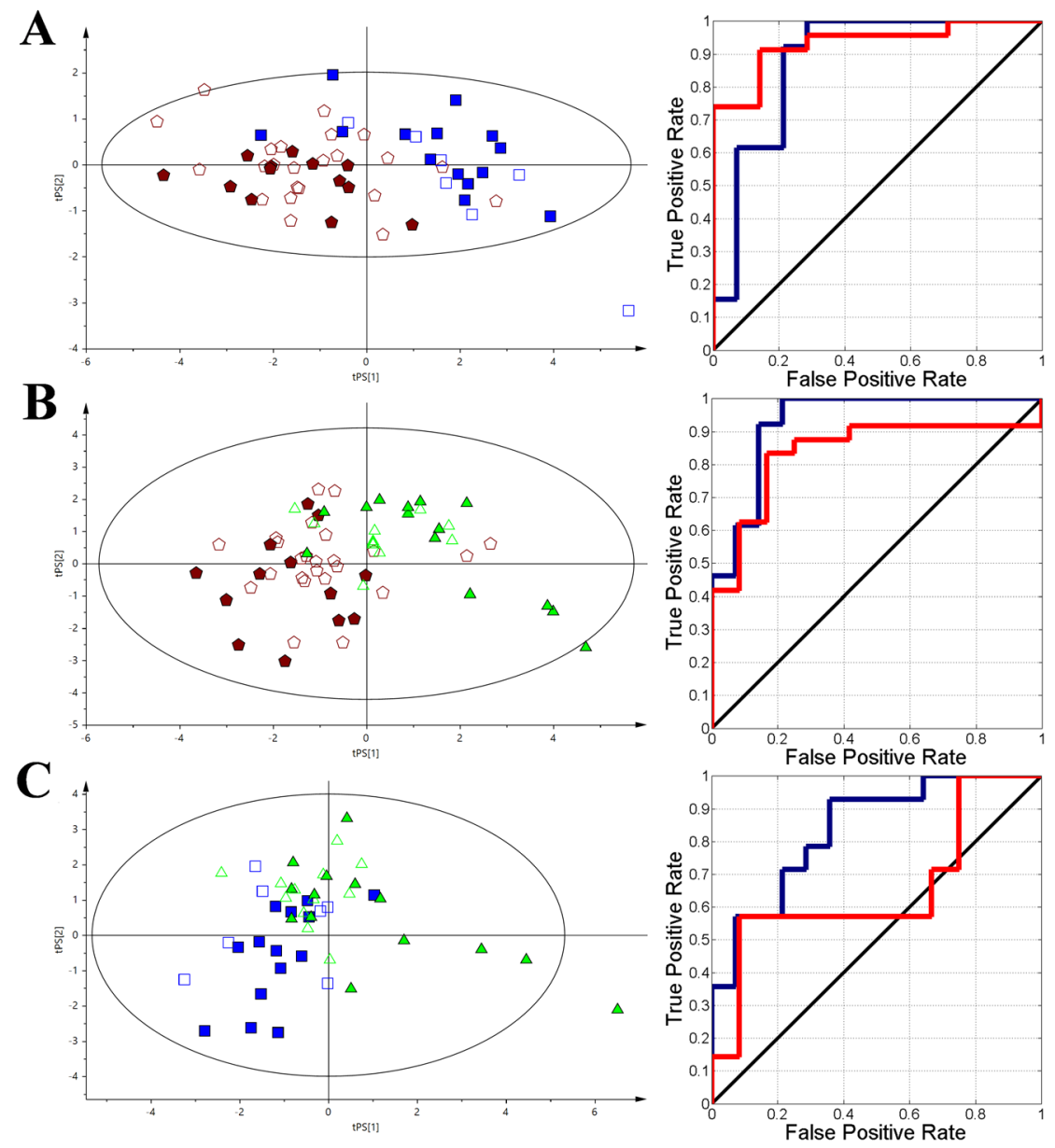

Figure 6: The VIP-PLS-DA model plots with the training (no fill) and test (fill) set samples and ROC curves. The dark blue curve represents the training set and the red curve represents the test set. (A) - AML vs CLL; (B) - AML vs nHL, (C) - nHL vs CLL. Red pentagon - AML; Green triangle - nHL and Blue box - CLL. 
treatment of these diseases is determined by early and accurate diagnosis. There are several cytologic, genetic and molecular tools used in diagnostic and monitoring hematological malignancies such as flowcytometry, FISH and PCR. Their sensitivity and clinical application however is heterogeneous.

In AML cytogenetic and molecular abnormalities are included in European Leukemia Net (ELN) prognostic classification [15]. The isolated mutation of NPM1 is related with favorable outcome whereas FLT3 ITD mutation is a poor prognosis factor. Recently,
Gerstung et al. showed that more personal approach is needed and combination of several driver mutations play important role in clinical course of the disease [16]. Prognosis in CLL patients with del $17 \mathrm{p}$ by FISH remains poor.

Both in AML and CLL another important tools for measurement of minimal residual disease (MRD) is flow cytometry with high sensitivity. There is several studies showing that progression-free survival (PFS) and overall survival (OS) in patients $\mathrm{MRD}(-)$ is longer compared to $\operatorname{MRD}(+)$.
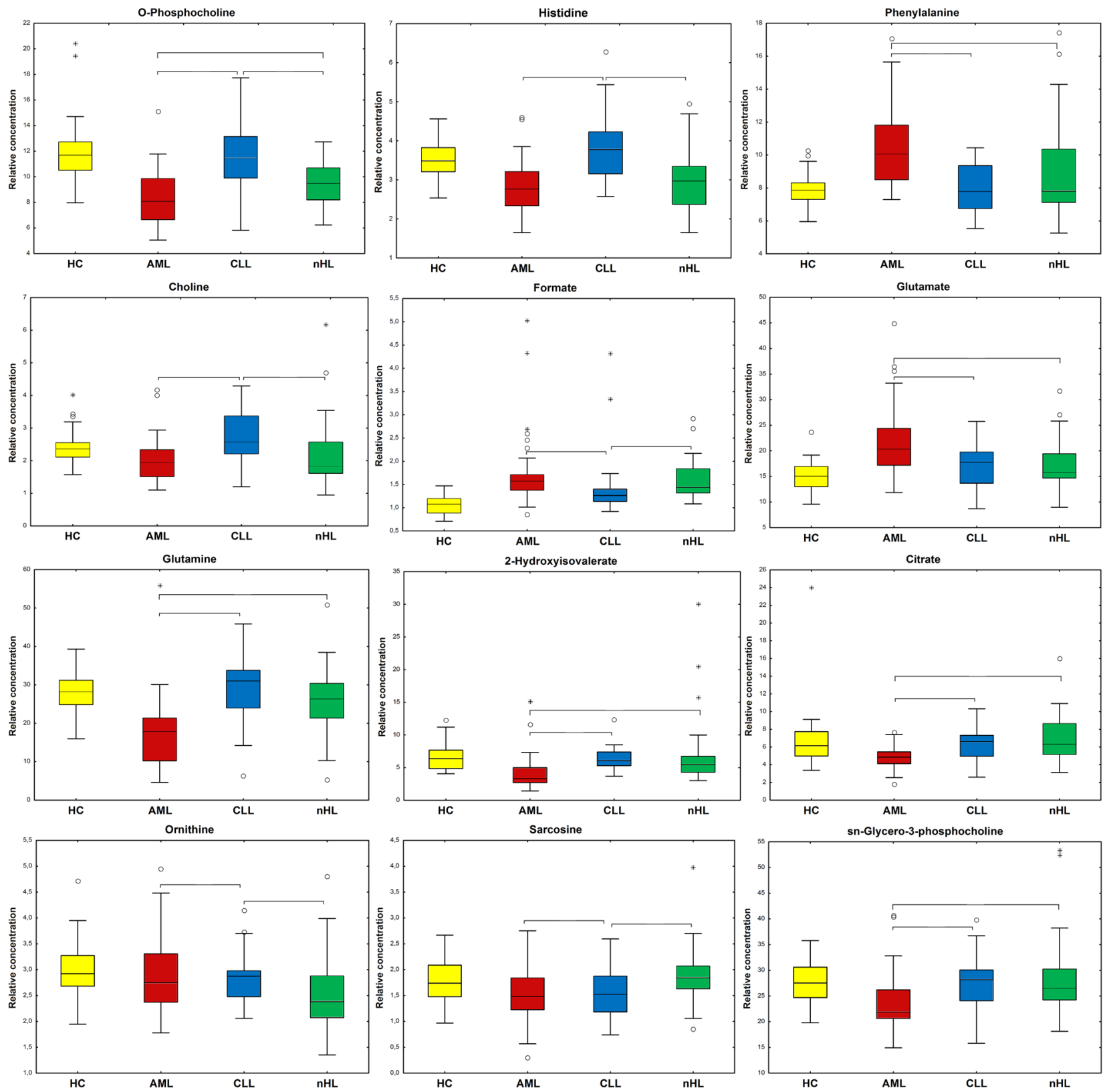

Figure 7: Boxplots for the identified statistically important metabolites $(p<0.05)$ between the haematological cancer groups. Braces show that the comparisons with the selected metabolites are statistically important. Whiskers - non-outlier min-max range; * - extreme; ${ }^{\circ}$ - outlier; bar - median; box - Q1-Q3 interquartile range; 
Most of methods mentioned need to be validated in prospective clinical trials. Moreover, introduction of new drugs - FLT3 inhibitors, BCR inhibitors, bcl2 inhibitors may abrogate prognostic value of cytogenetic and molecular abnormalities. Therefore more comprehensive approach including molecular and metabolomics tools may lead to personally tailoring cancer management.

Moreover, information about disease development could be valuable and supportive. The evaluation of changes in metabolite composition could be important for diagnosis, cancer stratification and as prognostic compounds with relevance for managing cancer therapies [17-20]. The objective of this study was to identify differences in metabolic changes between the healthy control group and the haematological malignancies groups with determination of the possible disturbances in the particular biochemical pathways. These findings might allow to better understand the nature of haematological disorders and enabling patient discrimination. Moreover, this approach could serve as supporting tool for earlier diagnoses and could lead to more suitable medical treatments.

According to acquired medical data (Supplementary Table 3) and clinical symptoms, AML is the most aggressive studied haematological malignancy. It causes the most visible changes to the serum metabolite profile ${ }^{1} \mathrm{H}$ NMR spectrum. This is reflected in the multivariate data analysis and statistics tests (Tables 1 and Supplementary Table 2, Figures 3 and 6). In nHL group less differentiating results in all data analysis was obtained, in comparison to AML, which also supports the trend of groups aggressiveness (Supplementary Table 3). Moreover, the CLL group compared to healthy control group also follow this trend and highlights more subtle than in the both cases -AML or nHL group vs HC (Table 1).

Nevertheless, it is evident that some of the metabolites were essential for all three haematological cancers (Supplementary Table 2).

The detailed analysis of the average survival time (Supplementary Table 3) showed that the studied haematological malignancies could have been sorted as follows: the most aggressive is AML (avg. $2.74 \pm 13.11$ months), followed by nHL (avg. $7.35 \pm 16.51$ months), and ending with CLL (avg. $12.61 \pm 13.40$ months). This observation has been supported by the quality of the chemometric models, specifically the most aggressive cancer revealed the best separation from the HC group and the best chemometrics model parameters (Table 1).

The results obtained in our study compared well with previously reported data in the literature $[17,19]$. The AML group is mostly consistent with overlapping in both studies statistically significant metabolites in the $\mathrm{HC}$ vs. AML comparison (Figure 3A and Supplementary Table 2), specifically the increase trends in phenylalanine in the AML group and the decrease in PC/GPC, choline, glutamine, and alanine were observed. The exception in our study (HC vs. AML) is the decrease in citric acid [17]. However, in another study based on GC-MS method, this metabolite was statistically significant and decreasing in the AML vs. HC comparison as in our study [19].

In literature there are not many scientific reports focused on investigating variations in low molecular weight compounds in nHL serum with general subgroups (Figure 3B and Supplementary Table 2) [21]. In this work all subgroups along with B lymphomas (with exclusion of CLL) were constituted one nHL group. Among the highlighted metabolites only phenylalanine matched with our study in the HC vs. nHL comparison [21]. In this study, this metabolite was increased for nHL but was not statistically significant. Moreover, only one literature reference is available and includes a metabolomics approach based on MALDI-TOF analysis of urine samples [22]. This publication highlights role of hypoxanthine as a main compound, which allows for nHL screening. The hypoxanthine levels in the $\mathrm{nHL}$ were significantly lower than in $\mathrm{HC}$ and permitted the differentiation of the selected groups. The finding obtained in our study shows highly elevated serum hypoxanthine (not statistically significant, VIP score $>$ 0.8 ) in the $\mathrm{nHL}$ group, which is opposite to the results obtained by B.C. Yoo et al. [22]. The differences in these two studies [22] could be associated with differences in used type of biological material [23].

In the comparison between the CLL and control group, our results only partially overlap with the literature data (Figure 3C and Supplementary Table 2) [18]. Previously reported outcomes by MacIntyre et al. highlighted increased pyruvate, glutamate, and proline concentrations and decreased isoleucine level. Our results, which rely on statistically significant metabolites, only matched with one compound - glutamate. The other metabolites were not statistically significant in our study, and only proline followed the same trend. In contrast, isoleucine was slightly increased in the CLL group. The main difference between literature data and this study was inability to detect a pyruvate signal for quantification, pyruvate was reported as the most important metabolite for separation in the mentioned work [18]. In context of this data, observed higher glucose levels in the CLL group could be related to a potential comorbidity of the high-age patient group [18]. Analysis of the co-morbidities in our study showed that 5 out of 25 patients in the CLL group and 3 out of 28 in the HC group could potentially have disturbances in glucose levels (Supplementary Table 4). Therefore, in our opinion a consequence of the raised glucose level, which was statistically significant in the $\mathrm{HC}$ vs. CLL comparison, could be directly associated with development of CLL, though this requires further verification.

In spite of both being haematological malignancies, the AML and CLL groups significantly differed from one another $[17,18]$. The alterations between the compounds 
in the different studies, which enabled their separation, could be caused by different disease stages or variability present in the taken control group. In case of CLL and nHL the separation was much less effective with coincides with the usual clinical presentation.

A recent study has reported that difficulties in cancer diagnosis could be due to the occurrence of co-morbidities in patients/healthy subjects and similar organism responses for different disease types [21]. It is known that additional factors related to immune system response may lead to more complex models that reflect the changes occurring in the cross-section of population [21]. Thus, it appears that important information from a diagnostic research could be obtained by comprehensive comparison of more than one disease with similar clinical symptoms or by having a more diverse control group.

\section{Biochemical insights into disease mechanism}

To focus on the most important metabolites that allowed for the differentiation of studied groups, metabolites obtained from the complex model (HC vs. AML vs. CLL vs. nHL) were adopted, even when the model parameters were not as good as in the detailed comparisons (between two groups) (Supplementary Table 2). The group of metabolites was selected based on the K-W test $(-\log 10(\mathrm{p})>4.00)$ and VIP analysis (VIP value $>1.00)$ and includes $\mathrm{O}$-phosphocholine, glutamine, phenylalanine, tryptophan, glutamate, histidine, formate, and 2-hydroxyisovalerate.

Throughout all the comparisons, $O$-phosphocholine appears to be one of most important metabolite, as it is widely considered to be a compound associated with the carcinogenic processes. $O$-phosphocholine is involved in glycerophospholipid metabolism, which is coupled to cancer cell metabolism and development [24]. Interestingly, this compound was considerably decreased in AML, displayed its lowest value among the haematological malignancies compared to the $\mathrm{HC}$ group. The second in $O$-phosphocholine level order in hetamological disorders is $\mathrm{nHL}$ and then CLL group with most similar value to $\mathrm{HC}$ (PC level $\mathrm{HC}>\mathrm{CLL}>\mathrm{nHL}>\mathrm{AML}$ ). The increased demand for glycerophospholipid metabolism compounds may be required for leukaemic cell proliferation. This suggests that the aggressiveness of the hematological diseases shows a decreasing trend among studied groups $($ AML $>$ nHL $>$ CLL) (Supplementary Table 3) $[25,26]$.

According to the list of the most influential metabolites examined in this study, glutamine was at the second position. In the literature, glutamine is reported as an essential compound for cancer development [27]. One of the most abundant uses of glutamine in biochemical pathways is its conversion to glutamate, which was also meaningful in our study. Glutamate is also transformed to $\alpha$-ketoglutarate and is involved in powering the TCA cycle. It was important that glutamine (CLL $>\mathrm{HC}>\mathrm{NHL}>$ AML) and glutamate (AML $>$ NHL $>$ CLL $>$ HC) showed complementarity or a negative correlation, in the relative integrals, between each other (Figure 7, Supplementary Table 2). This may indicate an extensive intensification in the glutamine-glutamate biochemical transformation. These two compounds might be also associated with haematological cancer aggressiveness and use of these metabolites in TCA cycle amplification accompanied by other cancer development processes. In the literature, glutaminase inhibition highlights possible negative effects of cancer progression by interrupting the glutamineglutamate pathway [28, 29]. Moreover, increased conversion of glutamine to glutamate may be essential for functioning of immune system cells [30].

Among amino acids, a decrease in tryptophan levels $(\mathrm{HC}>\mathrm{CLL}>\mathrm{AML}>\mathrm{nHL}$ ) could indicate increased tryptophan catabolism, which might be connected to degradation via the kynurenine pathway. This has been linked to local shutdown of the immune system response by indoleamine 2,3-dioxygenase (IDO) to promote malignant cell proliferation [31, 32, 33, 34]. Interestingly, the levels of the intermediate product - formate, is increased (Supplementary Table 2) and showed an opposing trend to tryptophan (AML $>$ nHL $>$ CLL $>$ HC). This finding could support the changes, that occur throughout the mentioned biochemical pathway by accelerating tryptophan breakdown to kynurenine [35]. The expression of the IDO enzyme was reported in the literature for the AML group [32]. Interestingly, the nHL groups had the most intense changes in tryptophan levels. Non-Hodgkin's lymphomas are a heterogeneous group of lymphoid malignancies that are usually present in the lymph nodes, spleen and bone marrow. nHLs, most often among studied units, can infiltrate tissue and organs, which could lead to many local changes and favourable immunosuppressive microenvironments. Recent research shown that utilization of an IDO inhibitor can be a promising agent in the treatment of nHL patients [36].

Another remarkable low molecular weight compound from the amino acids group in the haematological malignancies was phenylalanine. Its relative integral increased in all three haematological disorders groups $\mathrm{AML}>\mathrm{nHL}>\mathrm{CLL}>\mathrm{HC}$ and it could be related to protein breakdown. The BCAAs typically associated with this process are not statistically significant and do not cover the same order. Moreover, phenylalanine could amplify the requirement for fuelling tricarboxylic acid cycle for more aggressive haematological malignance through phenylalanine, tyrosine and tryptophan metabolism [37].

Our results showed that histidine negatively correlated with increased hematological diseases aggressiveness levels ( $\mathrm{CLL}>\mathrm{HC}>\mathrm{nHL}>\mathrm{AML}$ ), which could be connected with inflammation processes [38, 39]. The histidine metabolism pathway also participates in 
glutamate biosynthesis. Thus, the increased transformation of histidine into glutamate may be cause of lower level in haematological patients.

The identification of 2-hydroxyisovalerate levels (nHL $>$ CLL $>H C>A M L)$ in serum extracts of the haematological units was an unexpected observation, as it is most frequently assigned in urine as a potential indicator of lactic acidosis [40]. It was shown that lactic acidosis is a significant disturbance in the functioning of biochemical pathways in some haematological disorders studies [41, 42]. The main approach to determining the mentioned pathophysiological state was measuring lactate levels in blood. Compared to our results, elevated lactate levels were also observed in each unit, with the highest concentration visible in AML and nHL, which corresponds well with lactic acidic levels reported in the literature data [41, 42].

Considering the data originating from the general comparisons between the diseases (AML vs. CLL vs. nHL) (Figures 5D and 5E and Table 1) and beside the metabolites discussed earlier, we observed that 2-oxoisocaproate, citrate, sarcosine, ornithine, and taurine were the most important in second complex model.

2-oxoisocaproate (ketoleucine) is a shortchain keto acid, it along with other keto acids may be connected to kynurenine aminotransferase functioning as an amino group acceptor. However, its levels $(\mathrm{AML}>\mathrm{CLL}>\mathrm{nHL}>\mathrm{HC})$ were greatly increased in both of the leukaemia groups, though not in the lymphoma group (Supplementary Table 2) [43], which highlights the differences in the functioning of the selected haematological cancers.

The tricarboxylic acid cycle essential metabolite citrate (nHL $>\mathrm{HC}>\mathrm{CLL}>\mathrm{AML}$ ) had the lowest relative integral in AML among investigated groups, indicating the ability of increased TCA cycle functioning to be related to the increased activation of carcinogenesis processes. Despite the different values in the CLL and nHL groups, neither displayed the considerable citrate reduction observed in the AML group. This suggests that differences in the levels of this compound could be associated with the increased energy demand correlated with the rapid development of AML [19].

The metabolism of sarcosine ( $\mathrm{nHL}>\mathrm{HC}>\mathrm{CLL}>\mathrm{AML}$ ) leads to the rapid degradation to glycine, which demonstrates that serine and glycine metabolism may have an important impact on CLL and AML progression in contrast to $\mathrm{nHL}$, it could also lead to betaine metabolism [44, 45].

Ornithine (HC>AML $>C L L>n H L)$ is one of the main compounds in the urea cycle. The relative integral of this metabolite was lower in all three studied haematological cancers compared to the healthy control group. However, in the nHL samples, the ornithine levels differences were significantly higher than in the AML and CLL groups. Ornithine is mostly produced from arginine in the urea cycle, but could be also biosynthesized from proline and glutamine
$[46,47]$. In the literature, ornithine has been reported as an important agent for active T-cells. It can also lead to changes in biological pathways that involve ornithine usage and could be more prominent in nHL patients $[46,48]$.

The nHL group also had the lowest levels of taurine $(\mathrm{CLL}>\mathrm{HC}>\mathrm{AML}>\mathrm{nHL})$, and displayed significant differences from the other two studied groups. It has been reported that taurine could be early biomarker of tumour formation in breast cancer [49]. The literature data has reported that it is associated with a reduction of cancer development $[50,51]$. Therefore, the decreased taurine levels in the nHL group correspond to its extensive utilization for reducing malignant cell proliferation [52].

The described changes can be responses amplified by tumour cells as well as global reactions by the entire organism to pathological conditions influenced by a variety of metabolites. The overall interpretation of the changes in the compound levels in serum samples could be difficult due to fact that metabolites within their biochemical pathways may intertwine and overlap during homeostasis disturbances and changes directly caused by cancer cell metabolism.

Summing up discrimination of the studied cancers was possible based on the detailed chemometric calculation between healthy control and cancer groups as well as between them. The found list of compounds may be key to understand the metabolite biochemical changes occurring in selected cancers, but also potentially allow for the classification and discrimination of haematological malignancies, based on differences in small molecular compounds composition.

Obtained models parameters were connected with the clinical symptoms assumptions and increased aggressiveness of the haematological units (AML survival time avg. $2.74 \pm 13.11$ months, nHL avg. $7.35 \pm 16.51$, CLL avg. $12.61 \pm 13.40$ ), and amplified by the changes in the biochemistry of metabolites. The discrimination based on the metabolomics approach was greater for the more aggressive units, which seems to be logical. This assumption was confirmed by the visible correlation between the quality and significance of the chemometric models compared to the average survival time in the groups (AML $>$ nHL $>$ CLL).

Hypothetically, the obtained results showed that this metabolomics approach could allow for the additional verification of diagnosis or even faster assignment of individuals to specific cancers in the future. However more accurate and detailed biochemistry studies are required to understand the changes in metabolism and their functions in the selected haematological cancers.

\section{MATERIALS AND METHODS}

\section{Sample collection}

Peripheral venous blood samples were drawn from all the participants after overnight fasting for at least 8 hours. 
Blood samples were collected using Sarstedt S-Monovette system serum tubes (Sarstedt AG \& Co., Germany) that were centrifuged at $1000 \mathrm{x}$ g for 15 minutes at $4^{\circ} \mathrm{C}$. The serum samples serum were stored in 1,5 $\mathrm{ml}$ Eppendorf safelock tubes and maintained at $-80^{\circ} \mathrm{C}$ until analysis.

The study group included 116 patients with an established diagnosis of one of three different haematological malignancies. There were 38 patients with acute myeloid leukaemia (AML), 26 patients with nonHodgkin's lymphoma (nHL, CLL subgroup was excluded forming separate group) and 21 patients with chronic lymphocytic leukaemia (CLL). All the subjects were patients at the Haematology Clinic of Wroclaw Medical University in Poland. All recruited patients were in the active phase of the disease. All of the subjects had never been treated for their cancers. The serum samples were taken prior to the initiation of chemotherapy.

The control group consisted of 31 volunteers who were mainly recruited from familial doctors and from the Internal Diseases Clinic of Wroclaw Medical University in Poland. The volunteers were matched for age, sex, comorbidities (arterial hypertension, diabetes, ischaemic heart disease, and hypercholesterolemia) and smoking habits to the haematological groups. Baseline demographic and medical characteristics for the haematological groups and controls are presented in Supplementary Tables 3, 4 \& 5.

The study protocol was approved by the Bioethical Committee of Wroclaw Medical University (KB 41/2011) and each subject gave written informed consent.

\section{Sample preparation for proton NMR spectroscopy}

The collected serum samples were thawed at room temperature and vortexed. Then, $300 \mu$ of serum was transferred to a new Eppendorf tube and mixed with $700 \mu \mathrm{l}$ of cold methanol for protein precipitation. Next, the samples were homogenized (Qiagen, Tissuelyser LT) for $10 \mathrm{~min}$ at $50 \mathrm{~Hz}$ and then incubated for $20 \mathrm{~min}$ in $-20^{\circ} \mathrm{C}$. The homogenization step and incubation were then repeated. Subsequently, mixtures of serum-methanol were centrifuged for $30 \mathrm{~min}$ at $15000 \mathrm{rpm}$ at $4^{\circ} \mathrm{C}$. Afterwards, $700 \mu \mathrm{l}$ of supernatant was transferred to a new Eppendorf tube and then evaporated to dryness in a vacuum centrifuge (JWElectronic WP-03) for $5 \mathrm{~h}$ at 1500 $\mathrm{rpm}$ at $40^{\circ} \mathrm{C}$. The dry precipitate was dissolved in $600 \mu \mathrm{l}$ PBS (0.5 M, pH = 7.2, 20\% $\mathrm{D}_{2} \mathrm{O}$, and $330 \mu \mathrm{M}$ TSP) and then $550 \mu \mathrm{l}$ of the mixture was transferred to an NMR tube (SP, $5 \mathrm{~mm}$ ARMAR Chemicals). Samples were maintained at $4^{\circ} \mathrm{C}$ before the measurements were taken.

\section{${ }^{1}$ H NMR measurements}

The NMR spectra of the serum were recorded at $300 \mathrm{~K}$ using an Avance III spectrometer (Bruker, GmBH, Germany) operating at a proton frequency of $600.58 \mathrm{MHz}$.
The NMR spectra of the were recorded by using a cpmgldpr pulse sequence with water presaturation in Bruker notation. For each sample, 128 continuous scans were collected with a spin-echo delay of $400 \mu \mathrm{s} ; 80$ loops; a relaxation delay of $3.5 \mathrm{~s}$; an acquisition time of $2.73 \mathrm{~s}$; a time domain of $64 \mathrm{k}$; and a spectral width of $20.01 \mathrm{ppm}$. Two-dimensional NMR experiments (2D NMR) were recorded and processed for selected samples. Experiments performed included ${ }^{1} \mathrm{H}-{ }^{1} \mathrm{H}$ correlation spectroscopy (COSY), total correlation spectroscopy (TOCSY), and ${ }^{1} \mathrm{H}^{-13} \mathrm{C}$ heteronuclear single quantum correlation (HSQC). For the metabolomics workflow, the 1D spectra were processed with a line broadening of $0.3 \mathrm{~Hz}$, manually phased, baseline-corrected using the MestReNova software (Mestrelab Research v 11.0 ) and referenced to a TSP signal $\delta=0.0 \mathrm{ppm}$ for the serum samples. Methanol and water resonance signals were removed from the data matrix. All the spectra were normalized to a TSP resonance signal. The alignment of the resonance signals was completed via a correlation optimized warping algorithm (COW) [10] and the icoshift algorithm and implemented in MATLAB (v R2014a, Mathworks Inc.) [11].

\section{Pre-processing of variables for analysis}

A total 50 metabolites and 18 unknown resonance signals from the serum sample ${ }^{1} \mathrm{H}$ NMR spectra were assigned. The metabolite resonances were identified based on assignments published in the literature, the Chenomx software (v 8.2 Chenomx Inc.) and on-line databases (Biological Magnetic Resonance Data Bank (www. bmrb.wisc.edu) and Human Metabolome Data Base, (www.hmdb.ca). The NMR-measured metabolites were obtained as relative signal integrals of the non-overlapping resonance signals.

\section{Multivariate data analysis}

Multivariate data analysis was performed using the SIMCA software (v 14.0, Umetrics). The sample order in the dataset was randomized. All the variables were scaled to unit variance (UV), and the samples for model calculation were split into two sample sets (training and test) based on the Kennard and Stone algorithm. The discriminant version of the Partial Least Squares regression (PLS-DA) with a default $\mathrm{k}$-fold cross validation procedure was utilized to identify differences between the subgroups. To improve the obtained models, variable selection was conducted using VIP-plots with a confidence interval 0.95. Variables having VIP values below 0.8 were removed from the analysis. A single iteration was used to minimalize the overfitting models. New models were re-built based on selected variables, and their reliability were tested with a CV-ANOVA at a level of significance of $p<0.05$. The prediction performance of the VIP-PLSDA models was estimated based on receiver operating characteristic (ROC) curves and area under curve 
(AUC) values. For this purpose, a perfcurve function from the Matlab statistical tool-box (Matlab v. R2014a, Mathworks, Inc.) was adopted. Specificity and sensitivity were determined according to sample class prediction using the 7-fold cross-validated predicted values from the fitted $Y$-predev (implemented in SIMCA-14 software) for observations in the model.

\section{Pathway analysis}

The MetaboAnalyst 3.0 platform with selected features was used for metabolite data analysis. Pathway Analysis was performed on a relative integral matrix with only identified metabolites. All the data were scaled with Auto Scaling; the Pathway Enrichment Analysis was set on Global Test and the Pathway Topology Analysis was set on Relative-betweenness Centrality [12].

\section{Statistical data analysis}

The percent difference (PD) and relative standard deviation (RSD) for each assigned resonance signal were calculated. The percent difference was calculated based on the mean values of the metabolite's relative integral in each group. Data set with a relative integral were tested for the type of distribution based on the Shapiro-Wilk test. All assigned resonance signals were tested for statistical significance using an appropriate disturbance type test, either the Mann-Whitney-Wilcoxon or Student $t$ test using STATISTICA 12 (Statsoft Inc.). The K-W test was calculated for limitation to most influential metabolites in discus section. $\mathrm{K}-\mathrm{W}$ test was performed on the metaboanalyst platform for this purpose and all data were auto-scaled (http://www.metaboanalyst.ca) [12].

\section{Author contributions}

WW - performed the metabolomics experiment; data calculation; data analysis; substantive discussion; manuscript preparation;

$\mathrm{AC}$ - patient selection, substantive discussion

$\mathrm{AO}$ - data calculations

AZ - substantive discussion

$\mathrm{EP}$ - contributed reagents/materials/analysis tools, substantive discussion

JR, AB, MB, GM, TW, AS - patient selection, sample collection

PM - partial manuscript preparation; substantive discussion;

\section{Ethics approval and consent to participate}

The study was carried out in accordance with the Declaration of Helsinki. The study protocol was approved by the Bioethical Committee of Wroclaw Medical University (KB - 41/2011) and each subject gave written informed consent.

\section{Consent for publication}

All subjects read and signed a written informed consent prior the enrollment to the study.

\section{Availability of data and material}

The datasets generated during and/or analyzed during the current study are available from the corresponding author upon request.

\section{CONFLICTS OF INTEREST}

The authors declare that they have no competing interests.

\section{FUNDING}

The project was financially supported by NanoMat Project EIT+ POIG.01.01.02-02-002/08 and by Wroclaw University of Technology S40531/Z0303 together with WCB KNOW 2014-2018 grant.

\section{REFERENCES}

1. Tao J, Sotomayor E. Hematologic Cancers: From Molecular Pathobiology to Targeted Therapeutics. Springer Science+Business Media Dordrecht. 2012. ISBN 978-94-007-5027-2.

2. Gałązka-Sobotka M. Hematologia onkologiczna - aspekty kliniczne, ekonomiczne i systemowe. Warszawa. 2015. Uczelnia Łazarskieg. ISBN 978-83-64054-49-5.

3. Siegel RL, Miller KD, Jemal A. Cancer statistics, 2016. CA Cancer J Clin. 2016; 66:730.

4. Ferrara F, Schiffer CA. Acute myeloid leukaemia in adults. Lancet. 2013; 381:484-95.

5. Seyfried TN, Flores RE, Poff AM, D'Agostino DP. Cancer as a metabolic disease: implications for novel therapeutics. Carcinogenesis. 2014; 35:515-527.

6. Hirschey MD, DeBerardinis RJ, Diehl AME, Drew JE, Frezza C, Green MF, Jones LW, Ko YH, Le A, Lea MA, Locasale JW, Longo VD, Lyssiotis CA, et al. Dysregulated metabolism contributes to oncogenesis. Semin Cancer Biol. 2015; 35:S129-S150.

7. Brindle JT, Antti H, Holmes E, Tranter G, Nicholson JK, Bethell HW, Clarke S, Schofield PM, McKilligin E, Mosedale DE, Grainger DJ. Rapid and noninvasive diagnosis of the presence and severity of coronary heart disease using 1H-NMR-based metabonomics. Nat Med. 2002; 8:1439-4.

8. Deja S, Dawiskiba T, Balcerzak W, Orczyk-Pawiłowicz M, Głód M, Pawełka D, Młynarz P. Follicular Adenomas Exhibit a Unique Metabolic Profile. 1H NMR Studies of Thyroid Lesions. PLoS One. 2013; 8. 
9. Gowda GA, Zhang S, Gu H, Asiago V, Shanaiah N, Raftery D. Metabolomics-based methods for early disease diagnostics. Expert Rev Mol Diagn. 2008; 8:617-33.

10. Tomasi G, van den Berg F, Andersson C. Correlation optimized warping and dynamic time warping aspreprocessing methods for chromatographic data. J Chemom. 2004; 18:231241.

11. Savorani F, Tomasi G, Engelsen SB. Icoshift: A versatile tool for the rapid alignment of 1D NMR spectra. J Magn Reson. 2010; 202:190-202.

12. Xia J, Wishart DS. Using MetaboAnalyst 3.0 for Comprehensive Metabolomics Data Analysis. Curr Protoc Bioinformatics. 2016; 55:14.10.1-14.10.91.

13. Smith A, Roman E, Howell D, Jones R, Patmore R, Jack A; Haematological Malignancy Research Network. The Haematological Malignancy Research Network (HMRN): a new information strategy for population based epidemiology and health service research. Br J Haematol. 2010; 148:739-53.

14. Smith A, Howell D, Patmore R, Jack A, Roman E. Incidence of haematological malignancy by sub-type: a report from the Haematological Malignancy Research Network. Br J Cancer. 2011; 105:1684-92.

15. Döhner H, Estey E, Grimwade D, Amadori S, Appelbaum FR, Büchner T, Dombret H, Ebert BL, Fenaux P, Larson RA, Levine RL, Lo-Coco F, Naoe T, et al. Diagnosis and management of AML in adults: 2017 ELN recommendations from an international expert panel. Blood. 2017; 129:424-447.

16. Gerstung M, Papaemmanuil E, Martincorena I. Precision oncology for acute myeloid leukemia using a knowledge bank approach. Nat Genet. 2017; 49:332-340.

17. Wang Y, Zhang L, Chen WL, Wang JH, Li N, Li JM, Mi JQ, Zhang WN, Li Y, Wu SF, Jin J, Wang YG, Huang H, et al. Rapid diagnosis and prognosis of de novo acute myeloid leukemia by serum metabonomic analysis. J Proteome Res. 2013; 12:4393-401.

18. MacIntyre DA, Jiménez B, Lewintre EJ, Martín CR, Schäfer H, Ballesteros CG, Mayans JR, Spraul M, García-Conde J, Pineda-Lucena A. Serum metabolome analysis by $1 \mathrm{H}-\mathrm{NMR}$ reveals differences between chronic lymphocytic leukaemia molecular subgroups. Leukemia. 2010; 24:788-97.

19. Chen WL, Wang JH, Zhao AH, Xu X, Wang YH, Chen TL, Li JM, Mi JQ, Zhu YM, Liu YF, Wang YY, Jin J, Huang $\mathrm{H}$, et al. A distinct glucose metabolism signature of acute myeloid leukemia with prognostic value. Blood. 2014; 124:1645-54.

20. Karlíková R, Široká J, Friedecký D, Faber E, Hrdá M, Mičová K, Fikarová I, Gardlo A, Janečková H, Vrobel I, Adam T. Metabolite Profiling of the Plasma and Leukocytes of Chronic Myeloid Leukemia Patients. J Proteome Res. 2016; 15:3158-66.

21. Lindahl A, Forshed J, Nordström A. Overlap in serum metabolic profiles between non-related diseases:
Implications for LC-MS metabolomics biomarker discovery. Biochem Biophys Res Commun. 2016; 478:1472-7.

22. Yoo BC, Kong SY, Jang SG, Kim KH, Ahn SA, Park WS, Park S, Yun T, Eom HS. Identification of hypoxanthine as a urine marker for non-Hodgkin lymphoma by low-mass-ion profiling. BMC Cancer. 2010; 10:55.

23. Nielsen J, Jewett MC. Metabolomics: A Powerful Tool in Systems Biology. Springer Berlin Heidelberg. 2007. ISBN: 9783540747192.

24. Huang C, Freter C. Lipid metabolism, apoptosis and cancer therapy. Int J Mol Sci. 2015; 16:924-49.

25. Ridgway ND. The role of phosphatidylcholine and choline metabolites to cell proliferation and survival. Crit Rev Biochem Mol Biol. 2013; 48:20-38.

26. Glunde K, Serkova NJ. Therapeutic targets and biomarkers identified in cancer choline phospholipid metabolism. Pharmacogenomics. 2006; 7:1109-23.

27. Wise DR, Thompson CB. Glutamine addiction: a new therapeutic target in cancer. Trends Biochem Sci. 2010; $35: 427-33$

28. Jacque N, Bouscary D. Targeting glutamine uptake in AML. Oncoscience. 2014; 1:1-2. https://doi.org/10.18632/ oncoscience.1.

29. Jacque N, Ronchetti AM, Larrue C, Meunier G, Birsen R, Willems L, Saland E, Decroocq J, Maciel TT, Lambert M, Poulain L, Hospital MA, Sujobert P, et al. Targeting glutaminolysis has antileukemic activity in acute myeloid leukemia and synergizes with BCL-2 inhibition. Blood. 2015; 126:1346-56.

30. Newsholme P, Procopio J, Lima MM, Pithon-Curi TC, Curi R. Glutamine and glutamate - their central role in cell metabolism and function. Cell Biochem Funct. 2003; 21:19.

31. Masaki A, Ishida T, Maeda Y, Suzuki S, Ito A, Takino H, Ogura H, Totani H, Yoshida T, Kinoshita S, Narita T, Ri M, Kusumoto S, et al. Prognostic Significance of Tryptophan Catabolism in Adult T-cell Leukemia/Lymphoma. Clin Cancer Res. 2015; 21:2830-9.

32. Curti A, Aluigi M, Pandolfi S, Ferri E, Isidori A, Salvestrini V, Durelli I, Horenstein AL, Fiore F, Massaia M, Piccioli M, Pileri SA, Zavatto E, et al. Acute myeloid leukemia cells constitutively express the immunoregulatory enzyme indoleamine 2,3-dioxygenase. Leukemia. 2007; 21:353-5.

33. Uyttenhove C, Pilotte L, Théate I, Stroobant V, Colau D, Parmentier N, Boon T, Van den Eynde BJ. Evidence for a tumoral immune resistance mechanism based on tryptophan degradation by indoleamine 2,3-dioxygenase. Nat Med. 2003; 9:1269-74.

34. Le Dieu R, Taussig DC, Ramsay AG, Mitter R, MirakiMoud F, Fatah R, Lee AM, Lister TA, Gribben JG. Peripheral blood $\mathrm{T}$ cells in acute myeloid leukemia (AML) patients at diagnosis have abnormal phenotype and 
genotype and form defective immune synapses with AML blasts. Blood. 2009; 114:3909-16.

35. Brosnan ME, MacMillan L, Stevens JR, Brosnan JT. Division of labour: how does folate metabolism partition between one-carbon metabolism and amino acid oxidation? Biochem J. 2015; 472:135-46.

36. Nakamura N, Hara T, Shibata Y, Matsumoto T, Mabuchi R, Nakamura H, Kitagawa J, Kanemura N, Goto N, Shimizu M, Tsurumi H, Moriwaki H. Combination Of Indoleamine 2,3-Dioxygenase Inhibitor and Cytotoxic Agents Is a Novel Therapeutic Option For Non-Hodgkin Lymphoma. Blood. 2013; 122:4408.

37. Parker CE. Diseases of Phenylalanine Metabolism. Western Journal of Medicine. 1979; 131:285-297.

38. Niu YC, Feng RN, Hou Y, Li K, Kang Z, Wang J, Sun CH, Li $\mathrm{Y}$. Histidine and arginine are associated with inflammation and oxidative stress in obese women. Br J Nutr. 2012; 108:57-61.

39. Estey EH, Faderl SH, Kantarjian HM. Hematologic Malignancies: Acute Leukemias. Berlin: Springer Berlin Heidelberg. 2008.

40. Clarke JTR. A Clinical Guide to Inherited Metabolic Diseases 2nd edition. Cambridge University Press. 2002.

41. Sillos EM, Shenep JL, Burghen GA, Pui CH, Behm FG, Sandlund JT. Lactic acidosis: a metabolic complication of hematologic malignancies: case report and review of the literature. Cancer. 2001; 92:2237-46.

42. Chan FH, Carl D, Lyckholm LJ. Severe Lactic Acidosis in a Patient with B-Cell Lymphoma: A Case Report and Review of the Literature. Case Reports in Medicine. 2009; 2009:534561.

43. Han Q, Cai T, Tagle DA, Li J. Structure, expression, and function of kynurenine aminotransferases in human and rodent brains. Cell Mol Life Sci. 2010; 67:353-368.
44. Locasale JW. Serine, glycine and one-carbon units: cancer metabolism in full circle. Nat Rev Cancer. 2013; 13:572-83.

45. Ueland PM, Holm PI, Hustad S. Betaine: a key modulator of one-carbon metabolism and homocysteine status. Clin Chem Lab Med. 2005; 43:1069-75.

46. Wang R, Dillon CP, Shi LZ, Milasta S, Carter R, Finkelstein D, McCormick LL, Fitzgerald P, Chi H, Munger J, Green DR. The transcription factor Myc controls metabolic reprogramming upon $\mathrm{T}$ lymphocyte activation. Immunity. 2011; 35:871-82.

47. Phang JM, Pandhare J, Liu Y. The metabolism of proline as microenvironmental stress substrate. J Nutr. 2008; 138:2008S-2015S.

48. Diaz JI, Edinger MG, Stoler MH, Tubbs RR. Activated T-cell subsets in benign lymphoid hyperplasias and B-cell non-Hodgkin's lymphomas. The American Journal of Pathology. 1991; 139:503-509.

49. El Agouza IM, Eissa SS, El Houseini MM, El-Nashar DE, Abd El Hameed OM. Taurine: a novel tumor marker for enhanced detection of breast cancer among female patients. Angiogenesis. 2011; 14:321-30.

50. Tu S, Zhang X, Luo D, Liu Z, Yang X, Wan H, Yu L, Li H, Wan F. Effect of taurine on the proliferation and apoptosis of human hepatocellular carcinoma HepG2 cells. Experimental and Therapeutic Medicine. 2015; 10:193-200.

51. Zhang X, Tu S, Wang Y, Xu B, Wan F. Mechanism of taurine-induced apoptosis in human colon cancer cells. Acta Biochim Biophys Sin (Shanghai). 2014; 46:26172.

52. Mates JM, Segura JA, Alonso FJ, Marquez J. Sulphurcontaining non enzymatic antioxidants: therapeutic tools against cancer. Front Biosci (Schol Ed). 2012; 4:722-48. 